\title{
Abnormalities of horizontal gaze. Clinical, oculographic and magnetic resonance imaging findings. I Abducens palsy
}

\author{
A M Bronstein, Joan Morris, G Du Boulay, M A Gresty, P Rudge
}

\begin{abstract}
Fifty one patients with abnormalities of horizontal gaze were studied with magnetic imaging of the brain (MRI) and eye movement recordings to identify the loci of lesions responsible for isolated abducens palsy, conjugate gaze palsy and different types of internuclear ophthalmoplegias. The lesions responsible for a particular disorder were identified by overlapping enlarged drawings of the individual scans at comparable brainstem levels and identifying the areas where the abnormal MRI signals intersected. A statistical procedure was devised to exclude the possibility that the areas of overlap occurred by chance. In this paper, the findings in the group of patients with VI nerve palsy are reported since the location of their lesions could be predicted from known anatomy, so validating the procedure. The results were independently obtained with the overlapping technique and the statistical procedure and showed that the lesions were located in a region corresponding to the posterior part of the abducens fasciculus. This confirms that central lesions producing isolated lateral rectus weakness spare the abducens nuclei. The agreement between the procedures used and earlier clinical and experimental results suggest that the method we describe can be applied to locate the site of lesions on MRI scans in other groups of patients with more complex gaze disorders.
\end{abstract}

Medical Research Council NeuroOtology Unit, Institute of Neurology A M Bronstein M A Gresty P Rudge

Institute of Neurology G du Boulay

Computing and

Statistics Unit,

Institute of Neurology,

National Hospital,

Queen Square,

London

J Morris

Correspondence to: Dr A M Bronstein, MRC Neuro-Otolon, Neuro-Otology Unit, Institute of Neurology, National Hospital, Queen $3 \mathrm{~GB}$, United Kingdom.

Received 24 May 1989 and in revised form

4 September 1989

Accepted 18 September 1989

Most clinical studies relating abnormalities of horizontal gaze to CNS topography have been based on occasional case reports of brain stem strokes and tumours in which the lesions were visualised, either on CT scan or at necropsy. Frequently with these studies there were inadequate descriptions of the eye movement abnormality, either because of the severity of the underlying clinical condition or because these data were collected retrospectively and, more importantly, the radiological techniques used were inadequate for imaging brainstem lesions. ${ }^{1}$

In this and the following paper we report the findings in 51 patients with disorders of horizontal gaze in whom detailed clinical and oculo-graphic studies were made, together resonance imaging (MRI) scans of the brain. The advantages of MRI over other imaging methods of the brain stem and its value for identifying small brainstem abnormalities are well recognised and could be important in demyelinating disease, where eye movement disorders are frequent but in which the detectability of brainstem lesions by CT scanning is low..$^{2-5}$ However, the use of MRI to correlate abnormal function to CNS topography is limited by the presence of multiple or "silent" areas of abnormal MRI signal, a problem particularly important in demyelinating disease. For this reason it became clear that to localise lesions responsible for a particular eye movement disorder, it was necessary to develop a method capable of obtaining reliable clinical/ MRI topographic correlations.

Horizontal gaze defects can be divided into conjugate and disconjugate. The former include unilateral or bilateral gaze palsies and the latter include failure of abduction (VI palsy) or adduction (usually internuclear ophthalmoplegia). In this paper we will describe the findings in a group of patients with VI palsy of central origin. This group was dèliberately chosen as a starting point because of its relative clinical and anatomical simplicity enabling us to illustrate the technical procedure used and its statistical validation. The findings in the more complex types of gaze defects will be presented in the companion paper.

\section{Material and methods}

For convenience much of this section is common to the two papers. with the abnormalities found on magnetic
(A) Patients The 51 subjects of the study were classified on the basis of examination of their eye movement signs into VI nerve palsy, gaze palsy and internuclear ophthalmoplegia. If a patient presented a combination of disorders, they were classified according to the more prominent, for instance, a patient with a convergent paralytic squint due to a severe VI nerve palsy associated with some slight slowness of saccades in the yoked adductor will be unilateral gaze palsy.

There were seven patients (aged 18-39 years) with unilateral VI nerve palsy thought to be of central origin because of the presence of concurrent long tract or brainstem signs. Horizontal diplopia was a prominent symptom in all and each had limitation of abduction, varying classified as a VI nerve palsy and not as a 
from a few degrees to complete absence. Five patients had MS or a single brainstem episode that was probably demyelinating. One patient had a haematoma (patient 3) as an unexpected MRI finding and another had a pontine glioma (patient 1) (See fig 2A). An evaluation of the significance of MRI findings for clinical diagnosis in some of these patients has been reported elsewhere. ${ }^{26}$

(B) Ocular-motor assessment All patients had a full neurological and neuro-otological clinical investigation. Eye movements were recorded with bilateral monocular horizontal DC EOG on paper by means of an ink-jet polygraph (Mingograph). Monocular calibrations were obtained while the other eye was patched. In most patients vertical recordings were also made of the more mobile eye.

The targets for saccades consisted of a row of red LEDs subtending $10^{\circ}$ intervals at a distance of $50 \mathrm{~cm}$ from the nasum. Saccades were made from the centre LED to $30^{\circ}$ left and right, firstly 10 stimuli in one direction and then in the opposite; $20^{\circ}$ or $10^{\circ}$ were used if eye excursion was limited. The stimuli for eye movement were switching between the centre and the eccentric light. Head movement was restricted with a chin rest. The interval between stimuli was approximately one second but the main criteria for delivering a new stimulus was that the eyes had attained the target, as monitored on the paper trace. Hand measurements of peak saccadic velocity were made at a paper speed of $150 \mathrm{~mm} / \mathrm{s}$ and resolution of $1 \mathrm{~mm}=1^{\circ}$.

In addition, the following eye movement responses were also recorded: vestibulo-ocular reflex was tested in the dark with the subjects seated on a motorised Barany chair using horizontal angular velocity steps of $+1-40^{\circ} / \mathrm{s}$ and sinusoidal rotational stimuli with frequencies of $0 \cdot 1$ to $0.4 \mathrm{~Hz}$ and peak velocity of $40^{\circ} / \mathrm{s}$. Vestibulo-ocular reflex suppression was investigated during sinusoidal stimuli by having the subjects fixate an LED attached to and rotating with the chair, placed $50 \mathrm{~cm}$ from the nasum in line with primary gaze. Smooth pursuit was elicited with a laser target moving sinusoidally between 0.1 to $0.4 \mathrm{~Hz}, 20^{\circ}$ peak, and optokinetic nystagmus was evoked by a full field drum encircling the patient rotating at $+1-40^{\circ} / \mathrm{s}$. Patients were examined for spontaneous nystagamus in the light and dark in primary and on right and left gaze. A caloric test was performed according to the technique of Fitzgerald and Hallpike $^{7}$ with a modification suggested to investigate vestibulo-ocular reflex suppression. ${ }^{8}$

(C) Identification of lesions A 0.5 Tesla Picker superconducting system was used for all the MRI examinations in this study. During the period when observations were being collected several upgrades in radio frequency transmitter, frequency synthesiser and gradient and surface receiver coils resulted in progressive improvements in image quality.

Brain images were all obtained with the standard head receiver coil supplied by the manufacturer. Pictures from multislice imaging protocols in the transverse plane were available for all patients. The position of the head in the scanner typically maintained scan plane orientation at about $5-10^{\circ}$ positive to the orbito-meatal line. Eight or 16 transverse $10 \mathrm{~mm}$ thick slices with a $2 \mathrm{~mm}$ interslice separation were generally made, but towards the end of the study, software improvements allowed $5 \mathrm{~mm}$ contiguous slices to be made. In a few patients sagittal images were also available.

An imaging matrix of 128 by 256 was routinely employed, giving pixel dimensions of approximately $2.4 \mathrm{~mm}$ by $1.2 \mathrm{~mm}$. Each line of data collection was subject to two averages.

Spin-echo (SE) and inversion recovery (IR) sequences were used. The nomenclature for $S E$ and IR scans are SE TR/TE and IR TR/TI, the inversion time $\mathrm{TI}$, and the echo time $\mathrm{TE}$, and are defined in the glossary of NMR terms of the American College of Radiologists. ${ }^{9}$ On the $\mathrm{SE}$ sequences used, lesions appear as areas of increased signal (white) relative to normal brain. Most patients had SE $2000 / 60$ sequences, some early examinations varied from this, usually in the echo time (SE 2000/40). With IR sequences, usually IR $1500 / 500$, but sometimes IR 2000/500, lesions appear as low signal (black).

The images analysed were always those obtained in close temporal relationship with the oculographic examination. One observer (GduB) was unaware of the ocular-motor studies and identified and traced all the areas of abnormal signal. Doubtful areas were compared for confirmation in all the sequences (including IR) obtained from the patient on the same day and sought in neighbouring slices. A thin, smooth periventricular border of high signal in SE images, when of even thickness, has not been accepted as good evidence of the presence of an abnormality. Small areas of abnormal appearance seen in single slices and in only one sequence had to be very clearly delineated and far from possible confusion with CSF signal to have been accepted for inclusion.

Selective cuts of the scans were enlarged, normalised in size and drawn onto transparent acetate sheets using a projector. The transverse slice where the VIII nerves, internal auditory meatus and the labyrinth were visualised was identified in all patients. Due to the ascending course of the VIII nerves this cut represents a low pontine level, and for patients with VI palsy was the only cut drawn. In cases of gaze palsy, a cut in the region of the upper half of the pons was included and in cases of INO a third cut in the mesencephalon, in addition to the pontine slices, was drawn. The scans of patients with unilateral clinical signs were oriented as if they were right sided. The acetates were traced on a representative drawing of the brain stem at the appropriate level which thus hosted all the areas of abnormal signal from the different patients; for this procedure the IV ventricle, in particular its ventral border, the VIII nerves and internal auditory meatus and the brainstem-cerebellar contours, in that order, were used as anatomical 
reference points.

In this composite picture the areas where abnormal signals from different patients in the same clinically defined group overlapped could be seen. Of these, the areas where there was an overlap of the abnormal signals of at least half of the patients $\left(\mathrm{OL}_{50}\right)$ were considered likely to be the sites of the lesions responsible for the oculomotor signs common to the patient group. In the appendix a statistical procedure is described to test whether the overlaps could have occurred by chance.

The structures likely to be involved by lesions were identified by overlaying the acetates and $\mathrm{OL}_{50}$ drawings on to comparable brainstem sections to the same scale taken from neuro-anatomy atlases. ${ }^{11}{ }^{11}$ This procedure was done for each group of patients, using the $\mathrm{OL}_{50}$ drawings. In addition, estimates were made for each individual subject of the extent to which a structure intersected with an area of increased MRI signal as drawn on the acetates. Th:s was rated blindly by an observer on a scale of + to ++++ . The + signs relate both to the size of the area of abnormal signal with that of the anatomical structure investigated, providing an estimate of the percentage of area involved, and to the location of the lesion centrally or eccentrically with that structure; thus, a small lesion would be + in the centre of a larger structure and + - if placed eccentrically; a lesion about half the size of a structure would be ++ if located centrally and + if eccentric.

\section{Results}

Oto-neuro-ophthalmological results

Saccadic velocities compared with normal data from our laboratory ${ }^{12}$ are shown in table 1 . As expected, abduction velocities in the paretic eye were considerably reduced. In cases $1,5,6$

^Footnote: In the better images the medial and lateral lemnisci can be confidently identified and there are often recognisably differing regions of signal in IR scans that probably represen medial and lateral vestibular nuclei and VI nerve nuclei as well as the MLF. The IV nerve nucleus may also possibly be seen. This is not true however of poor quality scans and uncertainty remains about the precise margins of structures. Since the nucle and tracts of the brainstem cannot be more certainly identified than this by MRI, particularly on SE scans, the structures likely to be involved by lesions onere identified by the indirect procedure described above.

Table 1 Saccadic velocities to $30^{\circ}$ target steps in patients with VI nerve palsy

\begin{tabular}{|c|c|c|}
\hline \multirow[b]{2}{*}{ Patient } & \multicolumn{2}{|l|}{ Saccade Velocity $(\% / s)$} \\
\hline & To right & To left \\
\hline 1 & $\begin{array}{l}\text { RE not recordable }(W) \\
\text { LE } 320^{\star}(\mathrm{SGP})\end{array}$ & $\begin{array}{l}420 \\
325^{\star}\end{array}$ \\
\hline 2 & $\begin{array}{l}\text { RE } 510 \\
\text { LE } 594\end{array}$ & $\begin{array}{l}510 \\
290^{\star}(W)\end{array}$ \\
\hline 3 & $\begin{array}{l}\text { RE 200*(W) } \\
\text { LE446 }\end{array}$ & $\begin{array}{l}425 \\
383\end{array}$ \\
\hline 4 & $\begin{array}{l}\text { RE } 415 \\
\text { LE } 405\end{array}$ & $\begin{array}{l}420 \\
343(W)\end{array}$ \\
\hline 5 & $\begin{array}{l}\text { RE } 363 \\
\text { LE } 500\end{array}$ & $\begin{array}{l}356^{\star}(\text { SGP }) \\
270^{\star}(\mathrm{W})\end{array}$ \\
\hline 6 & $\begin{array}{l}\text { RE 232* (W) } \\
\text { LE 373* (SGP) }\end{array}$ & $\begin{array}{l}276^{\star} \\
320^{\star}\end{array}$ \\
\hline 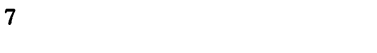 & $\begin{array}{l}\text { RE } 290^{\star} \text { (W) } \\
\text { LE } 355^{\star} \text { (SGP) }\end{array}$ & $\begin{array}{l}343^{\star} \\
315^{\star}\end{array}$ \\
\hline $\begin{array}{l}\text { Normal Controls }(n=25) \text {, } \\
\text { (mean and } 95 \% \text { confidence limits) }\end{array}$ & $\begin{array}{l}\text { RE } 453(324-582) \\
\text { LE } 504(381-627)\end{array}$ & $\begin{array}{l}529(389-669) \\
470(340-601)\end{array}$ \\
\hline
\end{tabular}

$(W)=$ direction of action of the clinically weak lateral rectus.

$\star$ = outside the $95^{\circ}$ \% confidence limits.

(SGP) = subclinical gaze palsy, velocity of the adductor yoked to the paretic lateral rectus below confidence limits. $\mathrm{RE}, \mathrm{LE}=$ right eye, left eye. and 7 there was also slowing of saccades in the direction of action of other muscles which was not evident on the initial clinical examination. Vertical saccadic velocities were normal in all but case 5 in which they were marginally slow (upwards $260^{\circ} / \mathrm{s}$, downwards $300^{\circ} / \mathrm{s}$; normal lower limit $396 \%$ and $342 \%$ respectively, $\mathrm{n}=12$ subjects age $20-38$ years). Six patients had asymmetrical vestibular responses in the form of directional preponderances of caloric or rotational nystagmus, measured as duration of the response, to the side opposite the lesion. This association between a central VI nerve palsy and a contralateral vestibular directional preponderance has not been recognised before. A detailed report and discussion of the vestibular findings, however, would not be relevant here and will be made separately. Directionality of other findings were not systematically related to the VI nerve palsy.

Location of the lesions producing VI nerve palsy All seven patients with VI nerve palsy had abnormal signals on the MRI at the lower pontine level and representative individual scans can be seen in fig 1 .

Figure 2A shows drawings of the abnormal signals of the seven cases. By comparing these with fig 2B, which illustrates some of the structures present in this area of the brain, it can be seen that in six cases the areas of abnormal signal were anterior, probably affecting the intra-brainstem fibres of the VI nerve (abducens fasciculus). In case 6 the abnormal signal was more posterior, close to the IV ventricle, and may have involved the VI nucleus to some extent. Table 2 shows the results of the individual abnormalities of MRI scans. In all patients there was involvement of the abducens fasciculus. Three out of the four patients with slowness of the adductor yoked to the paretic VI, constituting a "subclinical gaze palsy", also showed involvement of the VI nucleus and/or the nucleus reticularis pontis (NRP) caudalis. Those patients without a subclinical gaze palsy did not have VI nucleus involvement but one did have slight involvement of the NRP caudalis.

Figure 2C shows the area of the pons in which abnormal signals from different patients overlap. The $\mathrm{OL}_{50}$ (that is, the area with abnormal signal in four or more patients) encroaches upon the abducens fasciculus at the junction of the posterior and middle third portion of the fibres' course within the pons; the central tegmental tract, the facial nerve nucleus and the NRP caudalis are also involved.

Figure 3 illustrates the final stage of applying the statistical procedure described in the appendix. The shaded area in the matrix superimposed on the contours of the lower pons represents the only square where the significant level $(p<0.05)$ was achieved; that is, the probability that the overlaps in that square have occurred by chance is less than $5^{\circ}{ }_{0}$. The anatomical structures contained in that square would correspond to the abducens fasciculus and part of the surrounding NRP caudalis. 

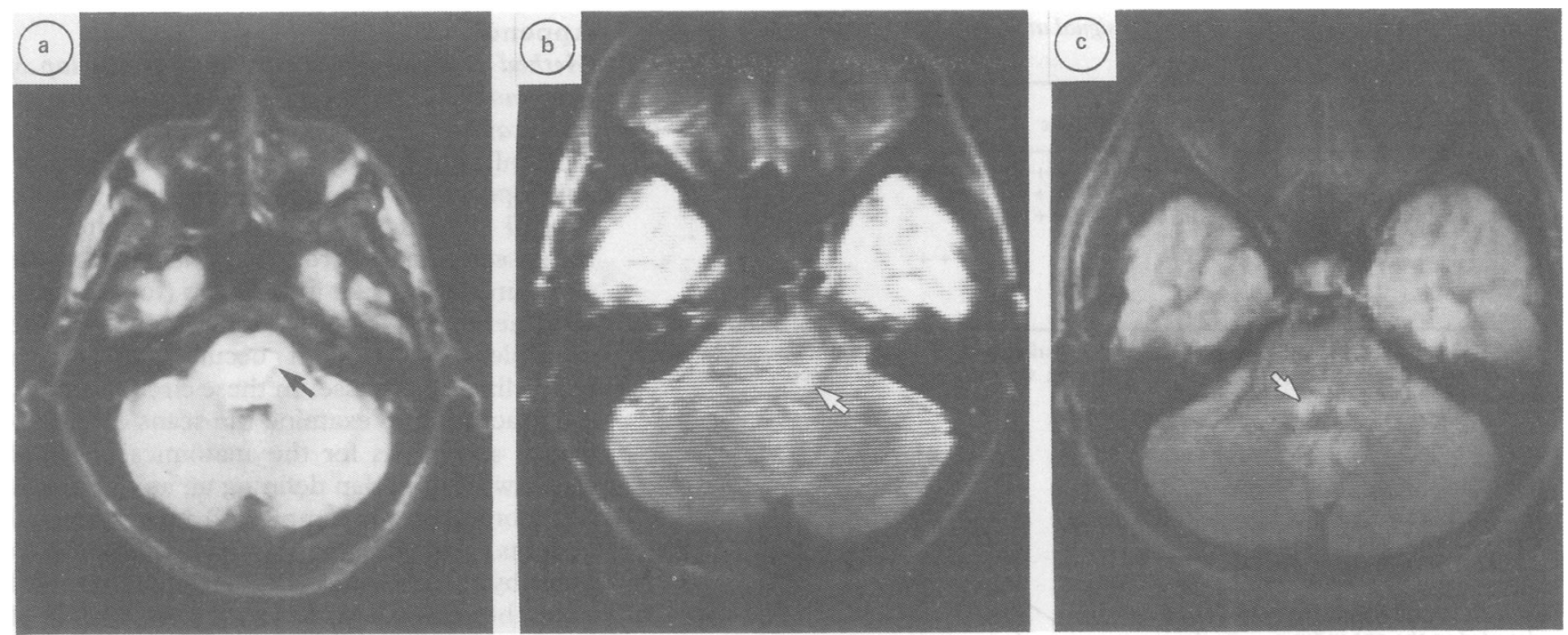

Figure 1 Representative transverse SE sequences at the low pontine level of three patients with unilateral abducens palsy. From left to right, patient 3 (Right sided VI palsy) with a pontine haematoma and patients 5 (LVI) and 6 (RVI) with acute brain stem episodes probably demyelinating in origin. In this and subsequent figures, the left side of the scan corresponds to right side of patients. The arrows point to the abnormal MRI signal.

\section{Discussion}

This study has shown that the lesion responsible for the central VI nerve palsy in our patients probably lies in the area of the posterior half of the abducens fasciculus. This conclusion is based on the results from the

Figure 2 a: Drawings of the areas with abnormal signal on MRI at the low pontine cut, in the seven patients with VI nerve palsy. All lesions were drawn as if they were right sided. $b$ : Significant structures present at the level of the low pontine cut.

AbdNr: abducens nerve

fasciculus. ML: medial

lemniscus. NRPC: nucleus reticularis pontis caudalis, the lateral and medial part are indicated. $M L F$ medial longitudinal fasciculus. IV: 4 th ventricle. NuPp: nucleus prepositus. AbdNu: abducens nucleus. SCP. superior cerebellar peduncle. VN: vestibular nuclei. ICP: inferior cerebellar peduncle.

FacNu: facial nucleus. FacNr: facial nerve fasciculus. CTT: central tegmental tract. The VIII nerves are also shown next to the emergence of the facial nerve.

$c:$ Low pontine structures as in fig $2(b)$. The hatched area is the $O L$ for the 7 patients with VI nerve palsy, that is, the common areas with abnormal MRI signal in at least four of the patients. identified $\mathrm{OL}_{50}$ areas and of the independent statistical procedure described in the appendix which are in remarkable agreement (compare figs $2 \mathrm{C}$ and 3 ).

The structure responsible for the generation of saccades and quick components of nystagmus has been termed the paramedian pontine reticular formation (PPRF) which, in animals, is located in the nuclei reticularis pontis (NRP) caudalis and oralis. ${ }^{13}$ Within the PPRF, excitatory "burst" neurons have been identified whose activity is directly related to saccade generation. These neurons undergo a sudden increase of discharge rate ("pulse") which is responsible for the high velocity of a saccade. ${ }^{14}$ The pulse is transmitted to the ipsilateral abducens nucleus exciting both abducens motor neurons and abducens interneurons. ${ }^{1617}$ The axons of these interneurons decussate immediately and ascend in the contralateral MLF to innervate the contralateral medial rectus neurons. ${ }^{1819}$ Thus, lesions of the VI nucleus produce paresis of abduction of the ipsilateral eye and of the yoked adduction of the contralateral eye, that is, an ipsilateral gaze palsy. ${ }^{20-22}$ Thus, it was not unexpected, in the light of this organisation, that in most of our cases the lesions with abducens palsy spared the VI nucleus. Only one patient (case 6 ) had abnormal signal in the area of the VI nucleus; the reasons why this patient did not have a full gaze palsy are unclear but it may be that the motor neuron in the abducens nucleus is more sensitive to injury than the interneurons.

The $\mathrm{OL}_{50}$ s extended on to non ocular-motor neighbouring structures, in particular the facial nerve nucleus and the central tegmental tract. The involvement of the VII is consistent with the frequent clinical association between VI and VII palsies in brainstem lesions. The involvement of the central tegmental tract may be at least partly responsible for ataxic features in these patients.

Comparison of individual lesions showed an interesting pattern. In those patients without associated subclinical gaze palsy the abducens fasciculus was the structure almost solely 
Table 2 Distribution of abnormal MRI signal at the low pontine level in patients with VI nerve palsy

\begin{tabular}{lllllll}
\hline Patient & $\begin{array}{l}\text { Sub Clinical } \\
\text { Gaze Palsy }\end{array}$ & VI Nu & VI Fasc & $\begin{array}{l}\text { NRPC } \\
\text { Lat }\end{array}$ & $\begin{array}{l}\text { NRPC } \\
\text { Med }\end{array}$ & MLF \\
\hline 1 & + & + & ++++ & ++++ & ++++ & +++ \\
2 & no & - & ++++ & - & - & - \\
3 & no & - & ++++ & - & - & - \\
4 & no & - & +++ & + & + & - \\
5 & + & + & + & +++ & + & - \\
6 & + & +++ & ++ & + & + & - \\
7 & + & - & \pm & - & - & \\
\hline
\end{tabular}

The + signs under a particular neural structure estimate their degree of intersection with an area of abnormal MRI signal $(++++=100 \%$; see the end of the material and methods section for a more detailed explanation).

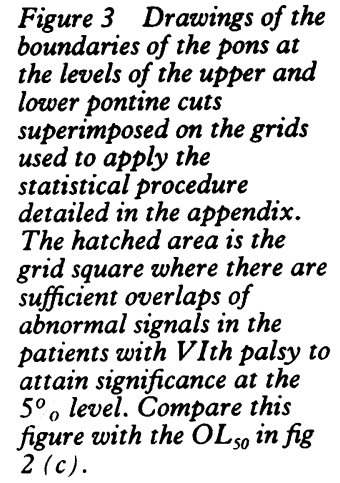

Figure 3 Drawings of the boundaries of the pons at the levels of the upper and lower pontine cuts superimposed on the grids used to apply the statistical procedure detailed in the appendix. The hatched area is the grid square where there are sufficient overlaps of abnormal signals in the patients with VIth palsy to attain significance at the $5^{\circ}{ }_{0}$ level. Compare this figure with the $O L_{s 0}$ in fig $2(c)$.
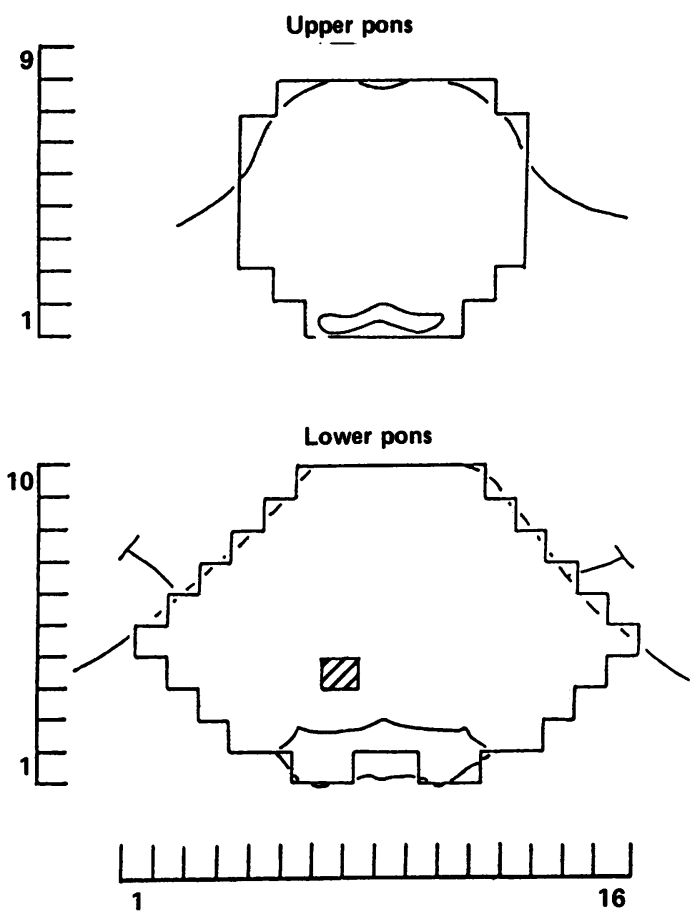

involved whereas in the presence of subclinical gaze palsy abnormal signals in the area of the NRP caudalis or the VI nucleus were present. For example, the abnormal MRI signal in patient 5, who had a subclinical gaze palsy, involved the lateral part of the NRP caudalis, thought to be part of the PPRF. ${ }^{17}$ However, the companion paper shows that cases with clinically evident rather than subclinical unilateral gaze palsy had lesions involving not only the area of the lateral NRP caudalis, but also reticular structures at higher levels in the pons such as the NRP oralis ${ }^{23}$. These observations suggest that the participation of the lateral NRP caudalis in saccade generation may not be crucial.

We believe that the agreement between the findings from $\mathrm{OL}_{50} \mathrm{~s}$, the statistical analysis and the current knowledge of the anatomical organisation of the abducens nerve, validates the method we have used to localise the areas of lesions on MRI scans responsible for a clinical sign common to a group of patients. This is exploited in the following paper ${ }^{23}$ concerned with more complex gaze disorders for which the sites of responsible lesions are less well established.

We are grateful to the Multiple Sclerosis Society of Great Britain and Northern Ireland for generous financial support and to Drs I Ormerod, D Miller, A Kermode and Mr D MacManus of the NMR Research Group at the National Hospital, Queen Square for their patient work in the collection of the MR images.
Appendix

Method to determine if the areas of overlap of abnormal MRI signals from different patients are significant

The following statistical technique has been developed to test the confidence with which a clinical sign may be related to the site of a common lesion in a group of patients who have a certain clinical sign in common (for example, VIth nerve palsy) but who, individually, have multiple lesions, as it occurs typically in demyelinating disease. In these circumstances, the practice is to examine the scans or pathological specimens for the anatomical areas of lesion which overlap defining an area which is common to the samples from individual patients. The problem is that overlaps may occur by chance and be unrelated to the clinical signs the patients have in common. This is of particular importance for MRI images in which areas of abnormal signal may be clinically silent. Accordingly, the method tests whether the number of lesions overlapping in a common area of brain within a group of patients is unlikely to have occurred by chance.

\section{Coding the Results}

The acetates drawn from the patients' scans were divided into grids (fig 3 ). The resolution of the grid corresponded to the area of the smallest abnormal signals drawn on the acetate (these in turn were determined by the smallest area of abnormal signal detectable on the MRI scans). Each square in the grid was coded with 0 or 1 , where: 0 indicates the absence of an abnormal signal; 1 indicates the presence of an abnormal signal. An abnormal signal was assumed to be present in a square if it covered more than $25 \%$ of the square.

These squares were rearranged into a row. The results were formed into a matrix, where each row was the results from a different patient and each column was a different square in the grid, that is, the results were organised as follows:

\section{$\mathrm{X}, \mathrm{Y}$}

square no: $1,1 \quad 1,2 \quad 1,3 \quad 2,1 \quad 2,2 \quad 2,3 \quad 3,1$ $\begin{array}{llllllll}\text { patient } 1 & 0 & 0 & 0 & 1 & 1 & 0 & 0\end{array}$

$\begin{array}{llllllll}\text { patient } 2 & 1 & 1 & 1 & 0 & 0 & 0 & 0\end{array}$

$\begin{array}{llllllll}\text { patient } 3 & 0 & 0 & 0 & 1 & 0 & 0 & 0\end{array}$

$\begin{array}{llllllll}\text { patient } 4 & 1 & 1 & 0 & 0 & 0 & 0 & 0\end{array}$

\section{NOTATION}

Let $C=$ Total number of squares in the grid

Let $R=$ Number of patients

Let $r_{i}=$ Total number of squares containing abnormal signals for patient $i . i=1$ to $\mathbf{R}$

Let $Z_{2}=$ Total over all possible pairs of patients of the number of squares where BOTH patients have an abnormal signal.

Let $Z_{3}=$ Total over all possible triplets of patients of the number of squares where ALL 3 patients have an abnormal signal.

And similarly for $Z_{4}, Z_{5}$ etc. 
Calculation of results

Assuming that the abnormal signals in each patient are equally likely to lie anywhere on the scan the theoretical means $\left(E\left(Z_{2}\right.\right.$, etc $\left.)\right)$ and variances of $Z_{2}, Z_{3}$ etc were derived ${ }^{2425}$ :

$E\left(Z_{2}\right)=\sum_{k=1}^{R} \underset{j<k}{r_{i} r_{j}}$

$\operatorname{Var}\left(Z_{2}\right)=\sum_{k=1}^{R}{ }_{j<k} \frac{r_{i} r_{j}}{C}+\frac{r_{i} r_{j}\left(r_{i}-1\right)\left(r_{j}-1\right)}{C(C-1)}-\frac{\left(r_{i} r_{j}\right)^{2}}{C^{2}}$

$E\left(Z_{3}\right)=\sum_{k=1}^{R} \underset{j<k i<j}{\frac{r_{i} r_{j} r_{k}}{C^{2}}}$

$\operatorname{Var}\left(Z_{3}\right)=\sum_{k=1}^{R} \frac{r_{j} r_{j} r_{k}}{C^{2}}+$

$\frac{r_{i} r_{j} r_{k}\left(r_{i}-1\right)\left(r_{j}-1\right)\left(r_{k}-1\right)}{C^{2}(C-1)^{2}}-\frac{\left(r_{i} r_{j} r_{k}\right)^{2}}{C^{4}}$

Similarly for $Z_{4}, Z_{5}$ etc

Using Chebyshev's Inequality the probability of the observed value of $Z_{2}$ (or greater) occurring is given by:

$\operatorname{Prob}\left(Z_{2} \geqslant=N\right) \leqslant=\operatorname{Var}\left(Z_{2}\right) /(N-m)^{2}$

where $\mathrm{m}=\mathrm{E}\left(\mathrm{Z}_{2}\right)$

If the probability of the observed value of $Z_{2}$ (or greater) occurring was greater than 0.05 , no further analysis was done, because it meant that it was reasonable to assume that the abnormal signals in each patient were equally likely to lie anywhere on the scan. If, however, this probability was less than 0.05 the null hypothesis of abnormal signals equally likely to lie anywhere was rejected.

Using Chebyshev's Inequality again, for the probability of $\mathrm{n}$ or more patients having an abnormal signal in at least 1 square to be less than $5^{\circ}{ }_{0}, n$ must be large enough to satisfy:

$\operatorname{Prob}\left(Z_{\mathrm{n}} \geqslant 1\right) \leqslant 0.05$

that is, Prob $\left(\left|Z_{n}-E\left(Z_{n}\right)\right| \geqslant 1-E\left(Z_{n}\right)\right)$ $\leqslant 0.05$

that is, $\operatorname{Var}\left(Z_{n}\right) /\left(1-E\left(Z_{n}\right)\right)^{2} \leqslant 0.05$

The matrix of results was then examined to see which, if any, squares had abnormal signals in at least $n$ patients. In this study the level of significance was chosen to be $95 \%$, however a $99^{\circ}$ o significance level (or any other value) could have been chosen by determining $\mathrm{n}$ so that the probability of $\mathrm{n}$ or more patients having an abnormal signal in at least 1 square was less than $1^{\circ} \%$.

\section{Comments}

In practice the areas significant at the $95 \%$ level of significance corresponded to areas on the scans where at least $50 \%$ of the patients had abnormal signals overlapping $\left(\mathrm{OL}_{50}\right)$ (fig 3 , also fig 3 in the companion paper). This agreement is a particular result of the combination of number of patients and abnormal signals and size of the abnormal signal and brain area examined. For instance if each patient had more abnormal signals they would be more likely to overlap. Therefore one would require more than $50 \%$ of the patients to have signals overlapping in a certain area for this area to be significant.

1 Henn V, Buttner U. Disorder of Horizontal Gaze. In: G Lennerstrand, D S Zee, E L Keller, eds. Functional Basis of Ocular Motility Disorders. Oxford: Pergamon Press, 1982:239-45.

2 Ormerod IEC, Bronstein A, Rudge P, et al. Magnetic resonance imaging in clinically isolated lesions of the brain stem. J Neurol Neurosurg Psychiatry 1986;49:737-43.

3 Bogousslavsky J, Fox AJ, Carey LS, et al. Correlates of brain stem oculomotor disorders in multiple sclerosis. Arch Neurol 1986;43:460-3.

4 Joseph R, Pullicino P, Goldberg CDS, Clifford Rose F. Bilateral Pontine Gaze Palsy. Arch Neurol 1985;42:93-4.

5 Atlas SW, Grossman RI, Savino PJ, et al. Internuclear Ophthalmoplegia: MR-Anatomic Correlation. $\mathrm{Am} J$ Neuroradiol 1987;8:243-7.

6 Ormerod IEC, Miller DH, McDonald WI, et al. The role of NMR imaging in the assessment of multiple sclerosis and
isolated neurological lesions. Brain 1987;110:1579-616.

7 Fitzgerald G, Hallpike CS. Studies in human vestibular Fitzgerald G, Hallpike CS. Studies in human vestibular
function: 1. Observations on directional preponderance function: 1. Observations on directional preponderance
(Nystagmusbereitschaft) resulting from cerebral lesions. (Nystagmusbereitschaft)
Brain 1942;65:115-37.

8 Hood JD, Korres S. Vestibular suppression in peripheral and central vestibular disorders. Brain 1979;102:785-804.

9 American College of Radiology, Subcommittee on NMR nomenclature and phantom development. Glossary of NMR terms. Magnetic Resonance in Medicine 1984;1: 414-3.

10 Olszewski J, Baxter D. Cytoarchitecture of the human brain stem. Reinhardt, Basel 1954

11 Haines DE. Neuroanatomy. An atlas of structures, sections and systems. Baltimore, Urban and Schwarzenberg; 1987.

12 Bird AC, Leech J. Internuclear ophthalmoplegia $\mathrm{Br} J$ Ophthal 1976;60:645-51.

13 Goebel HH, Komatsuzaki A, Bender MB, Cohen B. Lesions of the pontine tegmentum and conjugate gaze paralysis. Arch Neurol 1971;24:431-40.

14 Cohen B, Henn V. Unit activity in the pontine reticular formation associated with eye movements. Brain Res 1972;46:403-10.

15 Fuchs AF, Kaneko CRS, Scudder CA. Brainstem control of saccadic eye movements. Ann Rev Neurosci 1985;8: 307-37.

16 Highstein SM, Maekawa K, Steinacker A, Cohen B. Synaptic input from the pontine reticular nuclei to abducens motoneurons and internuclear neurons in the cat. Brain Res 1976;112:162-7.

17 Buttner-Ennever JA, Henn V. An autoradiographic study of the pathways from the pontine reticular formation the pathways from the pontine reticular formation
involved in horizontal eye movements. Brain Res 1976; involved in $155-64$.

18 Graybiel AM, Hartwieg EA. Some afferent connections of the oculomotor complex in the cat: an experimental study with tracer techniques. Brain Res 1974;81:543-51.

19 Highstein SM, Baker R. Excitatory termination of abducens internuclear neurons on medial rectus motoneurons: relationship to syndrome of internuclear opthalmoplegia. J Neurophysiol 1976;41:1647-61.

20 Carpenter MB, McMasters RE, Hanna GR. Disturbances of conjugate horizontal eye movements in the monkey. Arch Neurol 1983;8:231-47.

21 Meienberg O, Buttner-Ennever JA, Kraus-Ruppert $R$. Lesion of the abducens nucleus as the cause of a conjugate gaze paralysis. In: Lennerstrand G, Zee DS, Keeler EL gaze paralysis. In. Lennerstrand Le Rotility Disorders. Oxford: Pergamon Press, 1982;527-9.

22 Pierrot-Deseilligny $\mathrm{Ch}$, Goasguen J. Isolated abducens nucleus damage due to histocytosis X. Brain 1984; nucleus damag

23 Bronstein AW, Rudge P, Gresty MA, Du Boulay G, Morris J. Abnormalities of horizontal gaze. Clinical, oculographic and magnetic resonance imaging findings. II Gaze palsy and internuclear ophthalmoplegia. $J$ Neurol Neurosurg Psychiatry 1990;53:200-7.

24 Eicker PJ, Siddiqui MM, Mielke PW. A matrix occupancy problem. Annals of Mathematical Statistics 1972;43: 988-6.

25 Mantel N. Approaches to a health research occupancy problem. Biometrics 1974;30:355-62. 